\title{
Low-Power Optimized, Widely Customizable, Time-Controlled Wireless Sensor/Actuator Data Transmission with Commercial Off-The-Shelf Standard Components
}

\author{
Patrick K. Kroh ${ }^{1}$, Michael Fink ${ }^{1}$, Hans-Michael Günther ${ }^{1}$, Reinhard Lerch ${ }^{1}$ \\ ${ }^{1}$ Chair of Sensor Technology \\ Friedrich-Alexander-University Erlangen-Nuremberg \\ Paul-Gordan-Straße 3/5 \\ 91052 Erlangen, Germany \\ patrick.k.kroh@fau.de
}

\begin{abstract}
:
For common sensor/actuator applications in which signal data is only forwarded, ALOHA-protocol-based radio transmissions as well as full-stack radio solutions do not appear to be the most energy-efficient solutions. Thus, an own low-power and widely customizable radio solution has been developed. It is based on standard general purpose radio components and achieves high energy efficiency by use of time-controlled (TDMA) low duty-cycle transmissions. The presented solution is especially well suited to the task of periodic transmissions of measurement values. For demonstration, a sensor node has been implemented and the radio solution's performance has been evaluated.
\end{abstract}

Key words: Wireless, Sensor, Low-Power, TDMA, Standard Components

\section{Motivation}

Sensors are often deployed in remote positions as well as in places in which wired connections from sensors to a central controller are complicated to realize or even impossible. Examples include smart home sensors in existing buildings and sensors for moving parts of tooling machines. In these cases, the use of wireless sensors is mandatory.

In the above mentioned application areas, usually measurement values need to be sent to a controller regularly with a fixed-period low duty cycle and refresh intervals within the range of seconds and minutes.

Therefore, it is especially advantageous to maximize battery lifetime or even enable autonomous operation as this minimizes necessary maintenance which again leads to lower maintenance costs and also helps to avoid financial losses which result from machine downtimes for maintenance such as battery checking and changing.

Current low power radio solutions are often based on ALOHA or are full stack radio solutions. ALOHA-protocol-based solutions can show unfavorable energy efficiency due to possible packet collisions and the resulting collision resolutions. Full stack solutions, on the other hand, have got much protocol overhead and a rigid framework which limits customizability [1], [3], [6], [9]. Especially for the transmission of single measurement values, much of the mentioned overhead is unnecessary as no generic session handling is required in the targeted application cases.

We have been looking for a widely customizable and portable solution for our own low power and energy harvesting research. It had to be specifically tailorable to our current use cases, including specific timing and full control over the protocol. Thus, an own solution has been developed that is based on standard components.

\section{Concept}

The created solution is based on time-controlled bidirectional half-duplex communication and includes collision resolution [2], [4].

The main low power techniques that have been utilized, are: duty cycling, minimized protocol overhead, cross-layer-approach, minimized transmission/receive times, minimized packet lengths, maximized sleep times, interrupt-based programming paradigm, voltage scaling and use of dedicated hardware. 
In this context, cross-layer-approach means that the ISO/OSI-layer reference model has only been used for orientation, but that no stringent separation between the layers with buffers and overhead messages is implemented as this would cause additional computational overhead which would result in increased energy demand [7].

A star topology with a central master has been chosen as this enables simple and thus energy efficient addressing and scheduling (Fig. 1). Neither explicit routing, nor other management overhead is necessary.

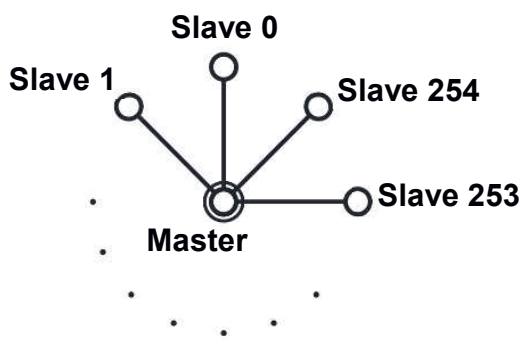

Fig. 1. Star topology with central master node.

Radio communication is time-controlled (TDMA) and based on an a priori schedule in order to avoid packet collisions, resulting collision resolutions and unnecessary idle times in receive mode which would all lead to higher energy demand [2], [10]. By use of a fixed schedule, the nodes have got the opportunity to maximize and optimize their individual sleep phases (Fig. 2). Fixed scheduling also ensures that compliance with duty cycle regulations for SRD and ISM frequency bands can be ensured. An ARQ ("Automatic Repeat Request") protocol is used for detection of packet losses [2], [4], [9]. Asynchronous messages are used for clock synchronization and can also be used for sporadic packet transmission. Clock drift is measured by the master node and is corrected on the fly. More information regarding the communication logic can be found in section "Protocol Logic".

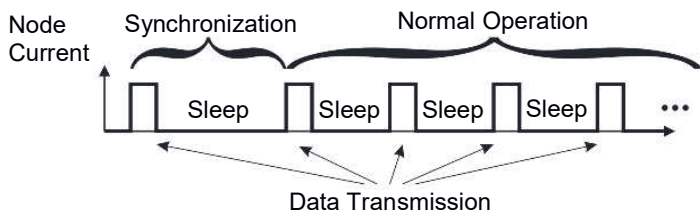

Fig. 2. Duty cycle operation.

The $868 \mathrm{MHz}$ frequency band has been chosen as there is less interfering radio traffic than in the commonly used $2.4 \mathrm{GHz}$ band and as the transmission power to range ratio is better as well [4].

The modulation scheme is binary FSK as it is relatively robust against amplitude distortions and because 2-FSK is usually supported by standard transceiver modules [4].

Other modulation schemes and frequency bands can be used for the implemented protocol if desired and may be part of future work.

The bit rate has been chosen to be $500 \mathrm{kbps}$, although different values can also be used (see also section "Demonstration"). As receive and transmission times shall be as short as possible, the bit rate needs to be chosen as high as possible. The desired maximum range has to be considered as well because necessary transmission power and susceptibility to distortions increase with bit rate at a given distance.

Another aspect is that utilization of standard components leads to good portability, simplified implementation and low hardware costs. No expensive ASIC development and manufacturing is thus required.

Theoretically, ultra-low power wake-up transceivers (ULPWUTs) can be used with the designed solution, too, as their deployment has also especially been considered during protocol design. Tests have not been performed yet.

\section{Protocol Logic}

The developed protocol can be classified as contention-free TDMA-based MAC-layer protocol if it had to be fitted into the ISO/OSIlayer reference model [10].

The time base for the scheduled communication is given by a real time clock (RTC). Slave nodes synchronize their RTCs to the master's one.

For synchronization between the master node and a slave node, one clock request message (CLKRQ) by the node is sufficient in the demonstration setup (Fig. 3). This property also contributes to the solutions' good energy efficiency.

Multiple CLKRQs can be used in order to compensate for significant clock deviation. This method has been tested, but was not necessary in the demonstration setup as all the nodes' RTCs worked accurately enough (oscillator: 20 ppm at $32.768 \mathrm{kHz}$ ) so that one CLKRQ sufficed for each node. Minor clock deviations are corrected on the fly by amendment of correction values to acknowledge packets. 


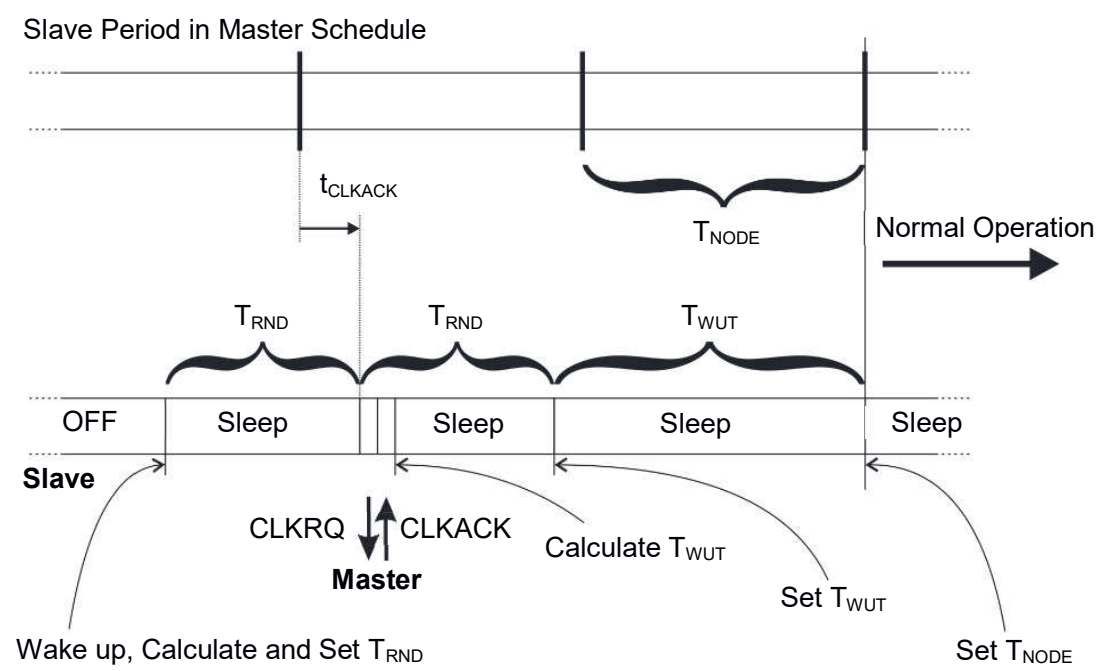

Fig.3. Clock synchronization.

Boundaries have been defined for the time durations from Fig. 3:

$t_{\mathrm{CLKACK}} \in \mathbb{N}_{0} \wedge t_{\mathrm{CLKACK}} \in\left[0 ; T_{\mathrm{NODE}}-1\right]$,

$T_{\mathrm{RND}} \in \mathbb{N} \wedge T_{\mathrm{RND}} \in\left[\frac{T_{\mathrm{NODE}}}{2} ; T_{\mathrm{NODE}}\right]$,

$T_{\text {WUT }} \in \mathbb{N} \wedge T_{\text {WUT }} \in\left[T_{\text {NODE }}+1 ; 2 T_{\text {NODE }}\right]$.

$t_{\text {CLKACK }}$ : time since last sensor period start

$T_{\text {NODE }}$ : sensor period

$T_{\mathrm{RND}}$ : random time

$T_{\text {WUT }}$ : sleep time until next properly scheduled wakeup

Choosing the boundaries as given, leads to a uniquely defined wakeup time of
$T_{\mathrm{WUT}}=2 T_{\mathrm{NODE}}-\left(t_{\mathrm{CLKACK}}+T_{\mathrm{RND}}\right) \bmod \left(T_{\mathrm{NODE}}\right) .(4)$

Acknowledge packets are used to detect packet losses in the manner of a Stop-and-Wait protocol. Lost packets are usually appended to the next scheduled measurement value transmission. It is also possible to schedule explicit packet resends, which may be beneficial if low refresh rates are used.

An a priori schedule is used in contrast to dynamic plug and play as the syncing and node registration that this would require, would also lead to additional energy demand. A communication example is shown in Fig. 4.

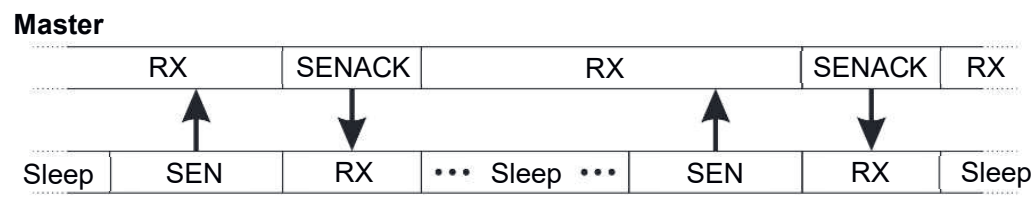

\section{Sensor Node}

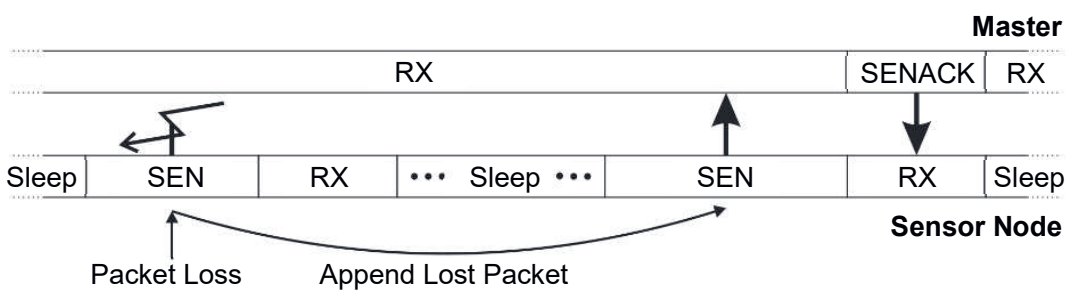

Fig.4. Measurement value transmission.

Transmitting measurement values and actuator commands asynchronously is possible as well and has also been included in the specification. For such transmissions, CSMA/CA ("Listen Before Talk") can be used to avoid collisions.
Actuator command transmissions work analogously to sensor node measurement value transmissions and are therefore not shown here in detail. The largest difference is that actuator nodes correct their own timing based on arrival 
times of packets from the master node, which they acknowledge.

The general protocol packet frame structure is depicted in Fig. 5. A packet consists of a preamble, a sync word, the payload and a CRC byte for error correction. There are different packet types for different purposes and each of those has got an individual payload structure. The most important types for the demonstration setup are CLKRQ, CLKACK, SEN, SENACK and SENACKCORR. Their structures are shown in Fig. 6.

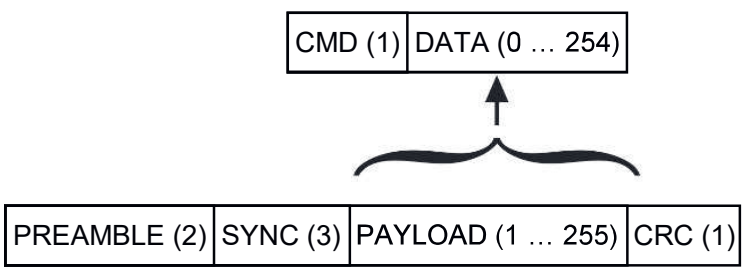

Fig. 5. General packet structure with field lengths in bytes.

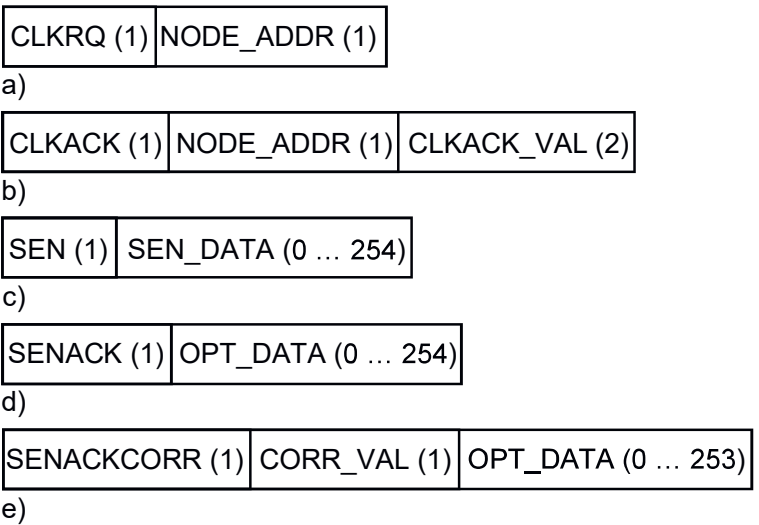

Fig. 6. Packet payload types with field lengths in bytes, (a) CLKRQ, (b) CLKACK, (c) SEN, (d) SENACK, (e) SENACKCORR.

- $\quad C L K R Q:$ clock request from slave node

- CLKACK: clock acknowledge from master node

- SEN: measurement values from sensor node

- SENACK: acknowledge from master for SEN packet

- SENACKCORR: acknowledge from master for SEN packet with included clock correction value for slave node's RTC

Each node has got an address of one byte length. The address is only sent as part of asynchronous messages as it is implicitly given for scheduled messages and can thus be omitted in these, which leads to shorter packet lengths and thus lowered energy demand. As there is one byte for the address, up to 255 slave nodes can be addressed by one master node, which itself has also got an address of its own. It is also possible to send broadcast and multicast messages if addresses are reserved for these. It must be noted that then consequently the number of addressable nodes is reduced.

\section{Demonstration}

The demonstration setup consists of four sensor nodes for temperature and relative humidity in air, one gateway/wireless master, one PLC and one Ethernet router as depicted in Fig. 7.

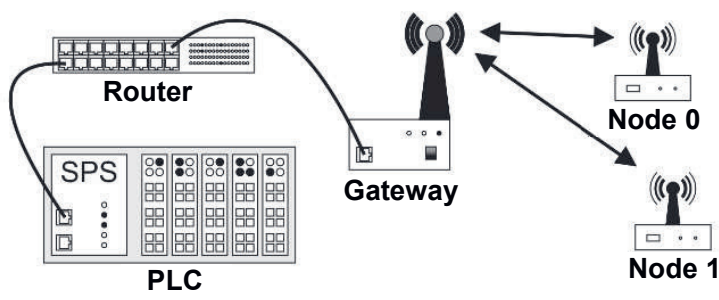

Fig. 7. Demonstration setup.

The following hardware was used for the sensor nodes: transceiver module STMicroelectronics (ST) SPSGRF-868, microcontroller ST STM32L063R8T6, temperature and humidity sensor HTU21D from TE Connectivity. Each node is powered by a CR2032 standard coin cell. Microcontrollers and radio modules are connected via SPI, the sensors are connected to the microcontrollers via I2C.

The gateway/wireless master is comprised of a microcontroller ST STM32F429ZIT6 and a radio module ST SPSGRF-868 which are connected via SPI.

The PLC controller is a WAGO $750-880$.

The wireless master serves as a gateway between the wireless and the Ethernet communication network. It works as TCP server and the PLC is a TCP client. Sensor data is buffered in the gateway and can be requested by the PLC when required. Additionally, other components on the network can be given access to the data, too.

The setup has been tested at different refresh rates. The standard rate is $1 \mathrm{~Hz}$ and more than $10 \mathrm{~Hz}$ have been tested with the simple setup.

Low power techniques that have been used with the microcontrollers, are: internal voltage scaling, power gating and clock gating. A sleep mode has been used in which register contents are kept. Use of even lower sleep modes is possible, but has not been programmed due to longer startup times from sleep and programming overhead regarding special register retention etc.

A further option for increased energy efficiency is the lowering of the supply voltage from $3.3 \mathrm{~V}$ 
to $2 \mathrm{~V}$ in order to achieve external supply voltage scaling, which may be used in future prototypes.

The achieved range has not been extraordinary in the current setup with about $10 \mathrm{~m}$ indoors. We assume that this is due to bad antenna gain and transmission power boost problems from the standard transceiver module and not a result from the set parameters. An indication for this assumption is that we were even unable to achieve a working link when we had correctly programmed the parameters of known working radio solutions such as the EnOcean Radio Protocol 2, which usually shows significantly larger wireless ranges than $10 \mathrm{~m}$ in commercial products [5].

The bit rate for transmissions has been set to $500 \mathrm{kbps}$. It is the highest rate that is supported by the deployed transceiver modules. Lower rates have also been tried in order to achieve greater link distances, but there was no recognizable difference. Hence, the highest possible bit rate has been chosen in order to minimize transmission/receive times and accordingly energy demand.

\section{Power Estimation}

The estimation of power demand and battery lifetimes for a sensor node is based on actual current and voltage measurements. For the measurements, an oscilloscope with a current probe (Rohde \& Schwarz RT-ZC20) and a voltmeter (Fluke 189) have been used.

A sensor node's power demand during sleep is $<6 \mu \mathrm{W}$ and consists of the power for the microcontroller and the radio module. The actual sensor's current can be neglected in comparison to the other two components (few nA).

The total energy per payload byte has been calculated to be in the range from $1.2 \mu \mathrm{J}$ to $142 \mu \mathrm{J}$ and depends on the number of bytes per packet and on the transmission power (here: $0 \mathrm{dBm}$ and $11.6 \mathrm{dBm}$ have been used).

The calculations include the time durations of microcontroller calculations and radio operation. It has been observed that most time and energy is spent on the SPI communication between microcontroller and radio module. Thus, in this demonstration, the largest power optimization potential lies in the reduction of the duration of inter-component communication.

As an example, the total energy for the transmission of four payload bytes at $11.6 \mathrm{dBm}$ is $146 \mu \mathrm{J}$ of which $107 \mu \mathrm{J}$ are caused by the microcontroller and $39 \mu \mathrm{J}$ by the transceiver module.

The total energy per payload byte depends strongly on the number of payload bytes as the constant energy demand from the overhead is much larger than the energy that is necessary for the radio transmission of a single byte.

Energy demand and accordingly battery lifetime mainly depend on refresh rate, number of payload bytes per packet, transmission power and battery type. They can be calculated approximately as follows:

$P_{\mathrm{AVG}}=\frac{P_{\mathrm{SLEEP}}\left(T_{\mathrm{NODE}}-T_{\mathrm{RUN}}\right)+P_{\mathrm{RUN}} T_{\mathrm{RUN}}}{T_{\mathrm{NODE}}}$,

$T_{\mathrm{BAT}}=\frac{E_{\mathrm{BAT}}}{P_{\mathrm{AVG}}}$.

$P_{\mathrm{AVG}}:$ Average power demand

$P_{\text {SLEEP }}$ : Power demand during sleep

$T_{\mathrm{RUN}}$ : Time in run mode for one period

$P_{\text {RUN }}$ : Power demand in run mode

$T_{\text {BAT }}$ : Battery lifetime

$E_{\text {BAT }}$ : Battery energy

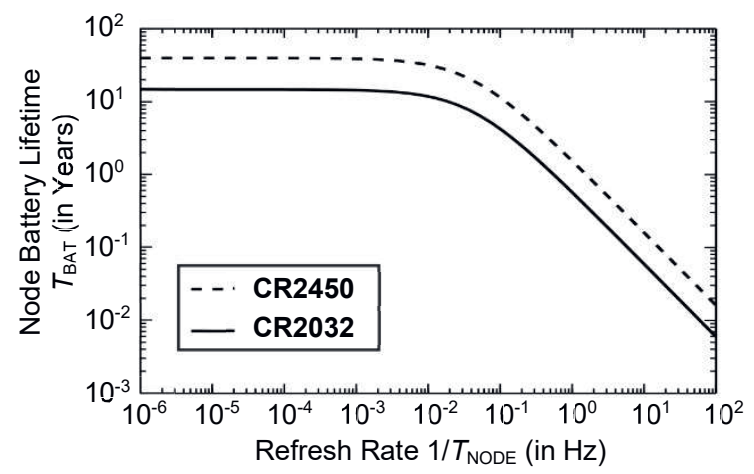

Fig. 8. Battery lifetime approximations with respect to sensor refresh rate.

In the given example with four payload bytes, a battery can last from 208 days (CR2032, 1 Hz refresh rate) up to more than 39 years (CR2450, refresh interval $>28$ mins) as demonstrated in Fig. 8. It can also be seen clearly that radio transmissions' influence on energy demand and thus battery lifetime is negligible at refresh intervals which are greater than 28 mins. At such low update rates, it is a sensor node's sleep current which dominates the power demand and thus has got the largest impact on battery lifetime.

It has to be noted that the present battery lifetime estimation is strictly theoretical as battery aging effects and leakage currents can have a significant influence and have not been included as these strongly depend on the actually used setup and environment. There is a strong dependence on PCB layout, environment conditions as well as battery type-dependent self-discharge. Battery self-discharge is about $1 \% / y e a r$ [Panasonic p.14] for ordinary coin cell 
batteries such as CR2032, but can also be lower for special long-term batteries such as BR2032 [8].

\section{Conclusion and Possible Future Work}

A fully customizable low power radio solution based on available standard components has been demonstrated. It shows low energy performance and enables optimization towards own specific applications. Various future work is possible and may be feasible depending on intended applications and environment conditions. An option is the inclusion of a pseudo-period for asynchronous transmissions into the schedule, resembling IEEE 802.15.4's superframe structure [6]. In addition, frequency hopping as well as Plug-and-Play with dynamic scheduling could also be used to enhance the solution. Another option is the use of different transceiver modules such as ULPWUTs and a further enhancement would be the use of multiple synchronized gateways for range enhancement as well as redundancy, and thus reliability improvement.

\section{References}

[1] Bluetooth SIG, BLUETOOTH SPECIFICATION Version 4.2., Bluetooth SIG (2014)

[2] J. Cecilio, Wireless Sensors in industrial timecritical environments, New York, Springer (2014), ISBN: 978-3-319-02888-0, DOI: 10.1007/978-3319-02889-7

[3] A. Dementyev et al., Power consumption analysis of Bluetooth, ZigBee and ANT sensor nodes in a cyclic sleep scenario, in: Wireless Symposium (IWS) (2013) IEEE International, [Piscataway, N.J.]: IEEE (2013), p. 1-4, ISBN: 978-1-46732141-9, DOI: 10.1109/IEEE-IWS.2013.6616827

[4] R. Gessler, T. Krause, Wireless-Netzwerke für den Nahbereich: Eingebettete Funksysteme: Vergleich von standardisierten und proprietären Verfahren, 2. aktualisierte u. erw. Aufl.

Wiesbaden: Springer Fachmedien Wiesbaden (2015), ISBN: 978-3-8348-1239-1, DOI: 10.1007/978-3-8348-2075-4

[5] D. A. Gratton, Introducing the EnOcean ecosystem, EnOcean Alliance (2016)

[6] M. Krauße, R. Konrad, Drahtlose ZigBeeNetzwerke, Wiesbaden, Springer Vieweg (2014), ISBN: 978-3-658-05820-3, DOI: 10.1007/978-3658-05821-0

[7] G. Miao, Cross-layer optimization for energyefficient wireless communications: A survey, in: Wireless Communications and Mobile Computing 9.4 (2009), p. 529-542, ISSN: 15308669. DOI: $10.1002 / \mathrm{wcm} .698$

[8] Panasonic, LITHIUM HANDBOOK, Hamburg, Panasonic (2015)
[9] M. Sauter, Grundkurs Mobile Kommunikationssysteme, Wiesbaden, Springer Vieweg (2015), ISBN: 978-3-658-08341-0, DOI: 10.1007/978-3-658-08342-7

[10] F. Xia, A. Rahim, MAC Protocols for CyberPhysical Systems, New York, Springer (2015), ISBN: 978-3-662-46360-4, DOI: 10.1007/978-3662-46361-1 